\title{
Alternative form of the Gelfand Levitan equation
}

\author{
Eric Kincanon * \\ Gonzaga University Spokane WA, USA \\ *Corresponding author E-mail: kincanon@gonzaga.edu
}

\begin{abstract}
This paper presents an alternative form of the Gelfand-Levitan Equation. By assuming a particular form of the spectral measure function and the potential kernel, an equation relating the potential and the reflection coefficient is found. This equation has an advantage over the Gelfand-Levitan Equation in that it can be solved without using iterative methods. The validity of the equation is demonstrated by looking at a singular and non-singular potential.
\end{abstract}

Keywords: Inverse Scattering; Gelfand-Levitan Equation; Reflection Coefficient; One-Dimensional Scattering.

\section{Introduction}

A potential $\mathrm{V}(\mathrm{r})$ can be determined from the reflection coefficient $\mathrm{R}(\mathrm{k})$ of a scattered wave using the Gelfand-Levitan equation [1-3]:

$K(r, s)+G(r, s)+\int_{-\infty}^{r} K(r, t) G(t, s) d t=0$

Where $\mathrm{G}(\mathrm{r}, \mathrm{s})$, the spectral measure function, is the Fourier transform of the reflection coefficient $\mathrm{R}(\mathrm{k})$ :

$\mathrm{G}(\mathrm{r}, \mathrm{s})=\frac{1}{2 \pi} \int_{-\infty}^{+\infty} \mathrm{R}(\mathrm{k}) \mathrm{e}^{-\mathrm{ik}(\mathrm{r}+\mathrm{s})} \mathrm{dk}+$ Bound State Terms

And $\mathrm{K}(\mathrm{r}, \mathrm{s})$ is related to the potential by:

$V(r)=-2 \frac{d K(r, r)}{d r}$

Finding V(r) is done by taking the Fourier transform of $R(k)$ to get $G(r, s)$ and then solving (1) for $K(r, s)$. Then (3) can be used to find the potential. The challenge arises in solving (1). This is typically done by successive iteration [4].

This paper first reviews this method of successive iteration, noting the challenges of the solution. Then an alternative solution to the Gelfand-Levitan Equation is presented. The validity of this new solution is then checked by considering a specific case.

\section{Gelfand-levitan equation solution by successive iteration}

To solve the Gelfand-Levitan Equation by using successive iteration, one assumes that $R(k)$ in equation (2) is known. Equation (2) then gives $G(r, s)$. Now, with $G(r, s)$ known the challenge is to find $K(r, s)$ in terms of $G(r, s)$. This is done by first rewriting equation (1) as:

$K(r, s)=-G(r, s)-\int_{-\infty}^{r} K(r, t) G(t, s) d t$

One can then use this equation to identify $\mathrm{K}(\mathrm{r}, \mathrm{t})$ as:

$K(r, t)=-G(r, t)-\int_{-\infty}^{r} K\left(r, t^{\prime}\right) G\left(t^{\prime}, t\right) d t^{\prime}$

Putting this expression in for $K(r, t)$ in equation (4) yields:

$K(r, s)=-G(r, s)+\int_{-\infty}^{r} G(r, t) G(t, s) d t-\int_{-\infty}^{r} G(r, t) \int_{-\infty}^{r} K\left(t, t^{\prime}\right) G\left(t^{\prime}, s\right) d u$

Equation (5) then is used again repeatedly on the right-hand side of (6) to give: 
$K(r, s)=-G(r, s)+\int_{-\infty}^{r} G(r, t) G(t, s) d t-\int_{-\infty}^{r} G(r, t) \int_{-\infty}^{r} G(t, u) G(u, s) d u+$ continued terms of iteration

Since $G(r, s)$ is known, the successive terms on the right hand side of equation (7) can be determined. One then hopes to find a convergence of the terms such that a general function can be found, or more commonly, that the terms become small enough that above a particular order they can be ignored.

The problem here is obviously the complexity of the right-hand side of equation (7). R(k) and, therefore, G(r,s) are typically not simple functions and even carrying out the integrals in equation (7) can be difficult. For many cases only the roughest of approximations can be obtained by this method.

\section{Alternative form of the Gelfand Levitan equation}

As an alternative to successive approximation, it is assumed that $K(r, s)$ and $G(r, s)$ have the forms:

$\mathrm{K}(\mathrm{r}, \mathrm{s})=\mathrm{g}(\mathrm{s}) \mathrm{F}(\mathrm{r})$

$\mathrm{G}(\mathrm{r}, \mathrm{s})=\mathrm{g}(\mathrm{s}) \mathrm{L}(\mathrm{r})$

Equation (4) can be rewritten as:

$G(r, s)=-K(r, s)-\int_{-\infty}^{r} K(r, t) G(t, s) d t$

Applying conditions (8) and (9) gives:

$g(s) L(r)=-g(s) F(r)-g(s) F(r) \int_{-\infty}^{r} g(t) L(t) d t$

Letting $s=r$ then gives:

$g(r) L(r)=-g(r) F(r)-g(r) F(r) \int_{-\infty}^{r} g(t) L(t) d t$

Using expressions (8) and (9) this can be rewritten as:

$G(r, r)=-K(r, r)-K(r, r) \int_{-\infty}^{r} G(t, t) d t$

There are two methods of solving this integral equation. The first method is to iterate equation (13) much as was done in the previous section. This yields:

$G(r, r)=-K(r, r)$

$+K(r, r) \int_{-\infty}^{r} K(t, t) d t$

$-K(r, r) \int_{-\infty}^{r} K(t, t) d t \int_{-\infty}^{t} K\left(t^{\prime}, t^{\prime}\right) d t^{\prime}$

$+K(r, r) \int_{-\infty}^{r} K(t, t) d t \int_{-\infty}^{t} K\left(t^{\prime}, t^{\prime}\right) d t^{\prime} \int_{-\infty}^{t^{\prime}} K\left(t^{\prime \prime}, t^{\prime \prime}\right) d t^{\prime \prime}$

+ successive terms

To simplify this, let $\mathrm{X}(\mathrm{t})$ be defined by:

$X(t)=\int_{-\infty}^{t} K\left(t^{\prime}, t^{\prime}\right) d t^{\prime}$

And so,

$K(s, s)=d X / d s$

Using (15) and (16), it is straightforward to show that:

$\int_{-\infty}^{r} K(t, t) d t \int_{-\infty}^{t} K\left(t^{\prime}, t^{\prime}\right) d t^{\prime}=X^{2} / 2 !$

Similar expressions can be found for the other terms of equation (14) giving:

$G(r, r)=-K(r, r)\left\{1-X(r)+\frac{X^{2}}{2 !}-\frac{X^{3}}{3 !}+\right.$ higher order terms $\}$

$=-K(r, r) \exp \{-X(r)\}$

$=-K(r, r) \exp \left\{-\int_{-\infty}^{r} K(t, t) d t\right\}$ 
Which can be rewritten as:

$G(r, r)=\frac{d}{d r} \exp \left\{-\int_{-\infty}^{r} K(t, t) d t\right\}$

Now using the relationships (2) and (3) gives:

$\frac{1}{2 \pi} \int_{-\infty}^{+\infty} R(k) e^{-i k(r+s)} d k=\frac{d}{d r} \exp \left\{-\frac{1}{2} \int_{-\infty}^{r} \int_{-\infty}^{s} V\left(t^{\prime}\right) d t^{\prime} d t\right\}$

This result gives a much more direct path relating the reflection coefficient, $\mathrm{R}(\mathrm{k})$, and the potential, $\mathrm{V}(\mathrm{r})$.

As an alternative method of deriving equation (20), equation (13) can be rewritten as:

$G(r, r)=-K(r, r)\left\{1+\int_{-\infty}^{r} G(t, t) d t\right\}$

This can further be rewritten as:

$-K(r, r)=\frac{d}{d r} \operatorname{Ln}\left\{1+\int_{-\infty}^{r} G(t, t) d t\right\}$

Solving for the bracketed term in equation (21) and applying that to this equation yields:

$-K(r, r)=\frac{d}{d r} \operatorname{Ln}\left\{-\frac{G(r, r)}{K(r, r)}\right\}$

Solving this for $\mathrm{G}(\mathrm{r}, \mathrm{r})$ gives:

$G(r, r)=-K(r, r) \exp \left\{-\int_{-\infty}^{r} K(t, t) d t\right\}$

Which agrees with the first method.

\section{Validity of alternative form}

To check the validity of equation (20), two potentials will be checked, one singular and one non-singular. As an example of a singular potential consider the delta function potential:

$V(r)=A \delta(r+\alpha)$

It is well known that the reflection coefficient for this potential is:

$R(k)=-i A \exp \{-2 i \alpha k\} / 2\left(k+\frac{i A}{2}\right)$

Putting this R(k) into the left-hand side of equation (20) and using contour integration gives:

$-\frac{A}{2} n(r+\alpha) \exp \left\{-\frac{A(r+\alpha)}{2}\right\}$

Where $n(r+\alpha)=1$ when $r \geq-\alpha$

$=0$ when $r<-\alpha$

Now considering the right-hand side of equation (20), one has:

$\frac{d}{d r} \exp \left\{-\frac{A}{2} \int_{-\infty}^{r} \int_{-\infty}^{s} \delta\left(t^{\prime}+\alpha\right) d t^{\prime} d t\right\}$

Which is easily shown to be in agreement with equation (26).

In order to complete the check of validity of equation (20), a non-singular potential need to be considered. For this purpose, one can look at the Eckart potential:

$V(r)=-2(b-a) \frac{e^{-b s}}{1+\beta e^{-2 b s}} \sinh (b s)$

$\mathrm{G}(\mathrm{r}, \mathrm{r})$ for this potential is given by Chadan and Sabatier [3] as:

$G(r, r)=(b-a) e^{-a r} \sinh (b r) / b$

Using expression (27) in the right-hand side of (20) gives this same result, thus completing the validity check of equation (20). 


\section{Conclusion}

Solving the Gelfand-Levitan Equation typically requires a tedious process of successive iteration. In addition, approximation methods are usually required. This paper presents an equivalent relationship between the reflection coefficient and the potential, as in the GelfandLevitan equation, that does not require the use of iteration. Examples of the validity of this relationship were given.

\section{References}

[1] I.M. Gelfand, B.M. Levitan, On the determination of a differential equation by its spectral function, Dokl. Akad. Nauk. USSR 77 (1951) 557-560

[2] I.M. Gelfand, B.M. Levitan, On the determination of a differential equation by its spectral measure function, Izv. Akad. Nauk. SSR 15 (1951) 309360.

[3] K. Chadan, P.C. Sabatier, Inverse Problems in Quantum Scattering Theory, Springer-Verlag, New York, 1977. https://doi.org/10.1007/978-3-66212125-2.

[4] R. Jost, W. Kohn, On the relation between phase shift energy levels and the potential, Danske Vid. Selsk. Math. Fys. 27 (1953) 3-19. 